\title{
Acidente Vascular Cerebral: Qual o Impacto nos Cuidadores Informais?
}

\section{Stroke: What is the Impact on Informal Caregivers?}

\author{
iD José Vítor Gonçalves ${ }^{1,2, *}$, Margarida Pereira ${ }^{3}$, João Capelo ${ }^{4}$, Joana Santos ${ }^{1}$, Filipa Vilabril ${ }^{5}$, Jorge Jacinto ${ }^{6}$ \\ 1-Serviço de Medicina Física e de Reabilitação / Centro Hospitalar Vila Nova de Gaia / Espinho, Portugal \\ 2-Faculdade de Medicina, Universidade do Porto, Porto, Portugal \\ 3-Centro de Reabilitação do Norte / Centro Hospitalar Vila Nova de Gaia / Espinho, Portugal \\ 4-Serviço de Reabilitação de Adultos 3 / Centro de Medicina de Reabilitação de Alcoitão, Portugal \\ 5-Serviço de Medicina Física e de Reabilitação / Centro Hospitalar De Trás-Os-Montes e Alto Douro, E.P.E., Portugal \\ 6-Diretor de Serviço de Reabilitação de Adultos 3 / Centro de Medicina de Reabilitação de Alcoitão, Portugal
}

DOI: https://doi.org/10.46531/sinapse/AO/200046/2021

\section{Resumo}

Introdução: Determinar o grau de sobrecarga dos cuidadores informais (Cls) de doentes internados por acidente vascular cerebral (AVC) em centro de reabilitação. Determinar quais as dimensões da vida dos $\mathrm{Cls}$ mais afetadas e determinar eventual relação entre: o grau de sobrecarga e a idade dos doentes, a idade dos $\mathrm{Cls}$, Medida de Independência Funcional (MIF) à admissão e alta, tempo decorrido desde o AVC e o de prestação de cuidados.

Material e Métodos: Durante Outubro e Novembro de 2019 foi proposto aos doentes avaliados em primeira consulta após alta a integração neste estudo. Foram recolhidos os dados sociodemográficos do doente e $\mathrm{Cl}$, determinado o grau de sobrecarga do $\mathrm{Cl}$ através do questionário de sobrecarga de Zarit (versão curta) e avaliadas eventuais relações entre variáveis através de análise estatística.

Resultados: Quarenta um doentes participaram e 22 tinham Cl (53\%). A maioria eram familiares em primeiro grau do doente e era a sua primeira vez como $\mathrm{Cl}$. A média e mediana de sobrecarga foi 17. Metade dos $\mathrm{Cl}$ apresentava níveis de sobrecarga no quartil superior da escala utilizada. Apenas existiu relação estatisticamente significativa na análise por clusters no intervalo interquartil para a MIF aquando da alta e o tempo decorrido desde o AVC.

Conclusão: Os resultados obtidos comprovam o alto desgaste a que os $\mathrm{Cls}$ são sujeitos pela prestação de cuidados em doentes previamente internados por AVC em centro de reabilitação. A ausência de maior número de relações estatisticamente significativas poderá estar relacionada com o baixo número da amostra.

\section{Abstract}

Introduction: Evaluate the burden of informal caregivers (ICs) of patients with stroke following discharge from rehabilitation center. Evaluate which dimensions of ICs's life are more affected and to determine an eventual relationship between the burden and the patients age, the ICs age, functional independence measure (FIM) at time of admission and discharge, time since stroke and time duration of care by the ICs.

Material and Methods: During October and November 2019 was proposed to all patients in the first appointment following discharge from rehabilitation center the participation in this study. Sociodemographic data from the patients and ICs were ob-
Informações/Informations: Artigo Original, publicado em Sinapse, Volume 21, Número 1, janeiro-março 2021. Versão eletrónica em www.sinapse.pt Original Article, published in Sinapse, Volume 21, Number 1 january-march 2021. Electronic version in www.sinapse.pt (C) Autor (es) (ou seu (s) empregador (es)) e Sinapse 2021. Reutilização permitida de acordo com CC BY-

NC. Nenhuma reutilização comercial.

(C) Author(s) (or their

employer(s)) and Sinapse 2021. Re-use permitted under CC BYNC. No commercial re-use.

Palavras-chave:

Acidente Vascular Cerebral; Cuidadores.

\section{Keywords:}

Caregivers;

Stroke.

*Autor Correspondente / Corresponding Author: José Vítor Gonçalves

R. Conceição Fernandes S/N, 4434-502 Vila Nova de Gaia, Portugal

josevitorrlg@gmail.com

Recebido / Received: 2020-09-02 Aceite / Accepted: 2021-01-23 Publicado / Published: 2021-04-16 
tained, the burden was determined by using the Zarit Overload Questionnaire (short version) and evaluated any relationships between variables by statistic analysis.

Results: Forty one patients agreed to participate and 22 had an IC (53\%). Most ICs were first-degree family members and it was their first time as IC. Average and median of the burden was 17. Half of the ICs presented a burden score in the higher quartile group of the used scale. There was a statistical significance relationship between the burden and the FIM at discharge and time since stroke in the interquartile statistic analysis.

Conclusion: The results obtained in this study verify the high burden in ICs of patients with stroke following discharge from rehabilitation center. The lack of more statistical significance relationships could be related to the low number of the sample.

\section{Introdução}

Em Portugal o acidente vascular cerebral (AVC) é a principal causa de morte e incapacidade.' A nível global e segundo a Organização Mundial de Saúde (OMS) o AVC vitimou 6 milhões de pessoas em 2016 sendo a segunda causa de morte em todo o mundo. ${ }^{2}$ A literatura é unanime em demonstrar que os sobreviventes apresentam inúmeras sequelas crónicas levando a uma diminuição na autonomia destes doentes e obrigando à presença de um cuidador para auxílio nas atividades de vida diária e na gestão das rotinas da vida após um AVC. ${ }^{3}$ A solução mais frequente no apoio a estes doentes passa pela presença de um cuidador informal $(\mathrm{Cl})$ no domicílio que não tem formação no que diz respeito às sequelas de AVC ou dos cuidados necessários/adequados para estes doentes. ${ }^{4} \mathrm{O} \mathrm{Cl}$ é a pessoa da rede social do doente, não remunerada, com relação significativa (familiar, parceiro (a), amigo(a) e/ou vizinho(a)), que se assume como o principal responsável pela organização, assistência e/ou prestação de cuidados. ${ }^{5}$ Se é verdade que estes cuidados são prestados por um membro da família na maior parte dos casos, ${ }^{6}$ não é menos verdade que o contínuo e necessário suporte prestado pelos cuidadores origina uma panóplia de consequências psicológicas, físicas, financeiras e sociais nos mesmos, colocando, por vezes, a assistência aos doentes em causa. ${ }^{7}$ Relativamente ao impacto e complicações sentidas pelos $\mathrm{Cls}$ começou a surgir na literatura o termo "sobrecarga" numa tradução de burden da literatura anglo saxónica. Este termo está relacionado com o conjunto de complicações a nível físico, psicológico e socioeconómico que surgem no decorrer do ato de cuidar ao longo do tempo. ${ }^{8}$ Neste contexto, o conceito de burnout apresenta-se como manifestação extrema desta sobrecarga / exaustão emocional e físi- ca. Este conceito envolve uma síndrome tridimensional como resposta a um evento crónico e com elevado grau de sobrecarga associado.' Inicialmente utilizado no ambiente laboral, foi denotado que também poderia ser aplicado em contexto da prestação de cuidados informais. ${ }^{10}$ Desta forma, e com o desenvolvimento de estudos posteriores passou a aplicar-se o conceito de burnout em muitos outros contextos como na relação parental ". O objetivo deste trabalho foi determinar qual - impacto e o grau de sobrecarga dos Cls de doentes previamente internados por AVC em centro de reabilitação através do preenchimento do Questionário de Sobrecarga Familiar de Zarit (QSFZ) - versão curta. Procedeu-se também à caracterização da população de doentes e dos respetivos Cls através da colheita dos dados sociodemográficos e procurou-se estudar a eventual presença de relações entre o grau de sobrecarga e as seguintes variáveis: a idade do doente, a idade do $\mathrm{Cl}$, o valor da medida de independência funcional (MIF) à admissão e data de alta, o tempo de prestação de cuidados do $\mathrm{Cl}$ (em meses) e o tempo decorrido (em meses) desde o evento vascular até à presente avaliação.

\section{Material e Métodos}

A população alvo deste estudo transversal consistiu em todos os doentes adultos observados pela primeira vez em consulta externa de reavaliação após alta de internamento por AVC em centro de reabilitação. Durante os meses de Outubro e Novembro de 2019 a todos os doentes observados que cumpriam estes requisitos foi proposto a participação neste estudo bem como ao respetivo $\mathrm{Cl}$ se presente. $\mathrm{A}$ ambos era solicitado e anexado no devido processo clínico a sua autorização através do consentimento informado para participação 
e análise dos dados recolhidos para este estudo estando garantido o seu anonimato em todas as fases do estudo. Este estudo foi também autorizado pela Comissão de Ética da instituição. A todos os doentes e Cls foram recolhidas as variáveis sociodemográficas, preenchido o QSFZ - versão curta e consultado o respetivo processo clínico relativo ao episódio de internamento. $O$ questionário em papel criado especificamente para este estudo foi posteriormente submetido na plataforma Google ${ }^{\circledR}$ Forms e foi depois exportado para o programa Microsoft ${ }^{\circledR}$ Excel 2016 sendo que a análise de dados para estudo de possíveis associações entre as variáveis foi realizada recorrendo ao programa SPSS ${ }^{\circledR}$, versão 26.0, Chicago, llinois, USA. Na avaliação desta sobrecarga do $\mathrm{Cl}$ podem ser utilizados diversos instrumentos sendo o mais utilizado o QSFZ (Burden Interview Scale) ${ }^{12}$ que é constituído por 22 questões. Existe também uma versão mais curta desta escala (QSFZ - versão curta) $)^{13}$ constituída por 12 questões em que cada questão pode pontuar entre 0 e 4 ( 0 - nunca, I - raramente, 2 - por vezes, 3 - muito frequente, 4 - quase sempre) e o resultado do questionário pode variar entre 0 e 48 sendo que valores mais elevados estão associados a maiores níveis de sobrecarga. Para esta escala existe uma distribuição do nível de sobrecarga segundo quartis com um primeiro quartil (QI) para valores totais de 3 , segundo quartil (Q2) para valores totais de 9 e terceiro quartil (Q3) para um valor total de $17 .{ }^{13}$ Através do cálculo do coeficiente de correlação de Spearman utilizando o SPSS ${ }^{\circledR} 26.0$ foi avaliada a eventual relação entre os níveis de sobrecarga e a idade do doente, a idade do $\mathrm{Cl}$, a MIF na admissão e na data de alta, o tempo de prestação de cuidados do $\mathrm{Cl}$ e o tempo decorrido desde o AVC. Esta análise estatística foi realizada inicialmente com toda a amostra e depois individualizada por clusters de sobrecarga. Tendo em conta que só existiu um doente com um grau de sobrecarga abaixo de QI esta segmentação foi apenas realizada para os outros dois grupos restantes.

\section{Resultados}

Durante os dois meses em que decorreu este estudo aceitaram participar $4 \mid$ doentes $(n=4 I)$ que cumpriam os critérios de inclusão com uma idade média de 62 anos. A etiologia em 28 doentes (70\%) foi AVC isquémico e em 12 doentes (30\%) AVC hemorrágico. A distribuição por sexos foi: 23 doentes (56\%) eram do sexo masculino e 18 doentes (43\%) eram do sexo feminino.
Relativamente à localização do AVC a circulação anterior do hemisfério esquerdo era a mais frequente em 28 doentes (70\%). À admissão o valor médio da MIF era de $72 / 126$ e à data de alta 86/ 126 sendo que, em média, os internamentos tiveram uma duração média de 60 dias. Relativamente a fatores de risco cardiovasculares à data do AVC: 29 doentes (71\%) realizavam terapêutica para hipertensão essencial, 15 doentes apresentavam diabetes mellitus sendo que apenas 3 eram insulino dependentes e os restantes realizavam terapêutica hipoglicemiante e 33 doentes (80\%) realizavam terapêutica para dislipidemia. Catorze doentes (34\%) eram fumadores ativos e 2 doentes (5\%) já tinham tido consumo de tabaco anteriormente ao evento vascular sem consumos ativos aquando do evento. Aquando da alta 29 doentes (70\%) estavam dependentes de apoio de terceira pessoa sendo que: 22 possuíam $\mathrm{Cl}, 5$ possuíam cuidadores remunerados, 2 estavam integrados na $\mathrm{RNCCl}$ (rede nacional de cuidados continuados integrados) e I estava integrado numa unidade residencial privada. Relativamente a estes 22 doentes com Cl: 5 doentes (23\%) integraram este estudo entre 3 a 6 meses após o AVC, 8 doentes (36\%) entre 6 a 12 meses, 3 doentes (14\%) entre 12 a 24 meses e 6 doentes (27\%) mais de 24 meses após data do AVC. Os dados relativos aos doentes com $\mathrm{Cl}$ encontram-se resumidos na Tabela 1 em anexo. Todos os doentes com $\mathrm{Cl}$ após internamento por AVC tiveram alta para o domicílio com o apoio do atual $\mathrm{Cl}$ como pode ser observado pela análise das Tabelas 1 e 2. Após a alta do internamento em centro de reabilitação a primeira consulta de reavaliação pós alta foi realizada em média 57 dias depois com os extremos de 40 dias e 68 dias como mínimo e máximo respetivamente. Relativamente aos $22 \mathrm{Cls}$ a idade média era de $5 \mathrm{I}$ anos sendo que $12 \mathrm{Cls}$ (55\%) eram do sexo feminino e $10 \mathrm{Cl}$ (45\%) eram do sexo masculino. Metade dos Cls eram Cls há menos de um ano. Quanto à escolaridade $8 \mathrm{Cls}$ apresentavam como grau de escolaridade o ensino básico, 9 Cls o ensino secundário e 5 Cls o ensino superior. Relativamente à situação profissional $9 \mathrm{Cls}$ estavam reformados, $7 \mathrm{Cls}$ mantinham a sua atividade profissional e $6 \mathrm{Cls}$ estavam desempregados sendo que 4 destes abandonaram a sua atividade laboral para exercer o papel de Cl. Dos $22 \mathrm{Cls}, 12$ eram cônjuges dos doentes, 9 eram pais ou filhos e em I dos casos o $\mathrm{Cl}$ era uma sobrinha. Os dados relativos aos Cls encontram-se resumidos na Tabela 2 em anexo. Nas respostas ao questionário de 
Tabela 1. Caracterização dos doentes que possuem cuidador informal.

\begin{tabular}{|c|c|c|c|}
\hline \multirow[t]{2}{*}{ Categorias de Dados } & \multirow[t]{2}{*}{ Variáveis } & \multicolumn{2}{|c|}{ Frequência } \\
\hline & & Absoluta (n) & Relativa (\%) \\
\hline \multirow[t]{2}{*}{ Género } & Feminino & 12 & 55 \\
\hline & Masculino & 10 & 45 \\
\hline \multirow[t]{4}{*}{ Tempo decorrido desde o AVC } & 3 a 6 meses & 5 & 23 \\
\hline & 6 a 12 meses & 8 & 36 \\
\hline & 12 a 24 meses & 3 & 14 \\
\hline & Mais de 24 meses & 6 & 27 \\
\hline \multirow[t]{2}{*}{ Tipo de AVC } & Hemorrágico & 6 & 27 \\
\hline & Isquémico & 16 & 73 \\
\hline \multirow[t]{2}{*}{ Localização AVC } & Hemisfério esquerdo (anterior) & 15 & 68 \\
\hline & Hemisfério direito (anterior) & 7 & 32 \\
\hline \multirow[t]{4}{*}{ Alteração da Linguagem } & Não & 17 & 77 \\
\hline & Afasia global & 3 & 13 \\
\hline & Afasia transcortical motora & 1 & 5 \\
\hline & Afasia de broca & 1 & 5 \\
\hline \multirow[t]{2}{*}{ Alteração da Fala } & Sim & 5 & 23 \\
\hline & Não & 17 & 77 \\
\hline \multirow[t]{2}{*}{ Quadro Neuromotor } & Hemiparésia & 21 & 95 \\
\hline & Tetraparésia & 1 & 5 \\
\hline \multirow[t]{2}{*}{ Disfagia } & Sim & 7 & 32 \\
\hline & Não & 15 & 68 \\
\hline
\end{tabular}

QSFZ - versão curta a média dos resultados dos $22 \mathrm{Cls}$ foi de 17 e a mediana foi de igualmente 17 a que corresponde ao valor do Q3. Nos extremos dos resultados encontra-se um valor máximo de 30 e um valor mínimo de I. Os dados relativos aos resultados de todos os $\mathrm{Cl}$ encontram-se na Fig. 1. Os resultados médios mais altos eram relativos a questões de dependência com um valor médio de I,82 e I,86 respetivamente. Já os valores médios mais baixos eram relativos a questões de receio / angústia com um valor médio de 0,82, 0,91 e I, 18 respetivamente. $O$ valor médio de todas as questões foi de 1,38 . Os quatro itens mais pontuados nos diferentes questionários foram: "Sente que, por causa do tempo que dedica ao seu familiar, não tem tempo suficiente para si próprio/a?"; "Sente-se stressado/a por ter de tomar conta do seu familiar e de tentar cumprir outras responsabilidades familiares ou profissionais?"; "Sente que deveria estar a fazer mais pelo seu familiar?"

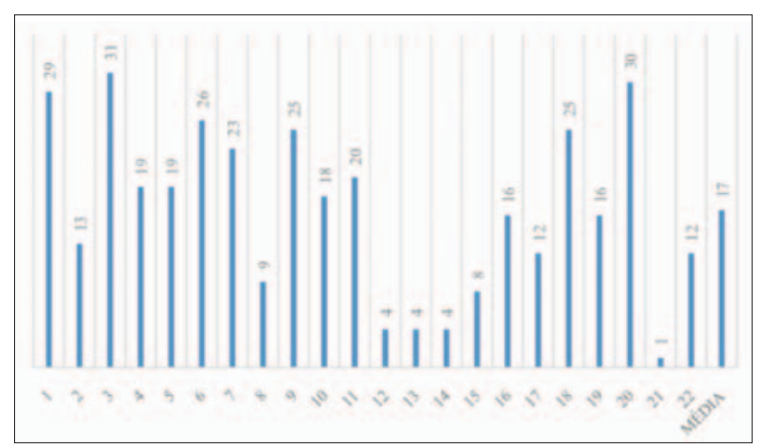

Figura 1. Distribuição do grau de sobrecarga por cuidador informal.

e, com igual pontuação média, "Sente que poderia fazer melhor ao tomar conta do seu familiar?". Os três itens menos pontuados foram: "Sente-se zangado/a quando está com o seu familiar?"; "Sente que o seu familiar prejudica presentemente os seus relacionamentos com outros elementos da família ou amigos?" e "Sente que 
Tabela 2. Caracterização dos Cuidadores Informais.

\begin{tabular}{|c|c|c|c|}
\hline \multirow[t]{2}{*}{ Categorias de Dados } & \multirow[t]{2}{*}{ Variáveis } & \multicolumn{2}{|c|}{ Frequência } \\
\hline & & Absoluta (n) & Relativa (\%) \\
\hline \multirow[t]{2}{*}{ Género } & Feminino & 12 & 55 \\
\hline & Masculino & 10 & 45 \\
\hline \multirow[t]{4}{*}{ Tempo de Cuidados } & 3 a 6 meses & 5 & 23 \\
\hline & 6 a 12 meses & 8 & 36 \\
\hline & 12 a 24 meses & 3 & 14 \\
\hline & Mais de 24 meses & 6 & 27 \\
\hline \multirow[t]{3}{*}{ Parentesco } & Cônjuge & 12 & 55 \\
\hline & Filhos & 9 & 41 \\
\hline & Outro familiar & 1 & 4 \\
\hline \multirow[t]{4}{*}{ Situação Laboral } & Reforma & 9 & 41 \\
\hline & Trabalhador ativo & 7 & 32 \\
\hline & Baixa & 2 & 9 \\
\hline & Desemprego & 4 & 18 \\
\hline \multirow[t]{2}{*}{ Abandono atividade laboral } & Sim & 4 & 18 \\
\hline & Não & 18 & 82 \\
\hline
\end{tabular}

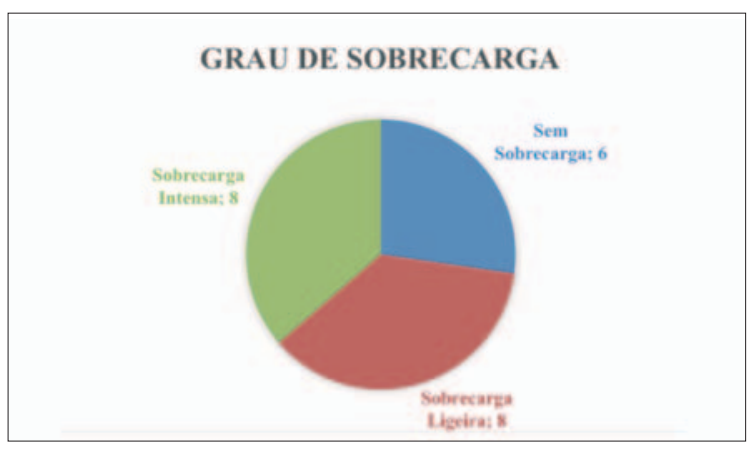

Figura 2. Grau de sobrecarga global dos cuidadores informais.

não dispõe de tanta privacidade como gostaria de ter por causa do seu familiar?". Nos extremos do valor total por cada questão obtivemos um valor total de $4 \mathrm{I}$ na seguinte questão "Sente-se stressado/a por ter de tomar conta do seu familiar e de tentar cumprir outras responsabilidades familiares ou profissionais? "e o valor mais baixo 18 na seguinte questão: "Sente-se zangado/a quando está com o seu familiar?". $\mathrm{Na}$ análise da relação entre as diferentes variáveis e os níveis globais do grau de sobrecarga não foram encontrados resultados estaticamente significativos. Existiu apenas uma associação positiva entre as diferentes variáveis e o grau de sobrecarga com exceção dos valores de MIF em que a associação foi negativa. Relativamente aos níveis globais de sobrecarga verificou-se a seguinte distribuição entre quartis: um $\mathrm{Cl}$ com um valor total de sobrecarga abaixo do QI, dez Cls com um valor total de sobrecarga na amplitude interquartil (entre QI e Q3): quatro acima de Q2, um igual a Q2 e cinco acima de Q2. Onze Cls apresentavam um valor total de sobrecarga acima do Q3. Relativamente à análise da relação entre variáveis por clusters de sobrecarga no grupo de Cls entre QI e Q3 foi encontrada uma associação negativa ( $r$ =$0,632)$ estatisticamente significativa $(p=0,050)$ entre a MIF aquando da alta o grau de sobrecarga e uma associação positiva $(r s=0,642)$ estatisticamente significativa $(p=0,045)$ entre o tempo decorrido desde o AVC e o grau de sobrecarga. Relativamente à relação entre variáveis no grupo de Cls acima de Q3 não foram encontrados resultados estatisticamente significativos.

\section{Discussão}

Neste estudo a idade média dos doentes (62 anos) foi inferior à idade média descrita na literatura para a ocorrência do AVC. ${ }^{14-16}$ Apesar de alguns estudos defenderam uma diminuição da idade média para a ocorrência 
do $\mathrm{AVC}^{15,17}$ a maioria dosAVC continua a ocorrer numa população com uma idade superior a 65 anos. No entanto, o facto de se tratar de um centro de reabilitação poderá contribuir para este resultado pelo enviesamento da referenciação de doentes mais novos com AVC em virtude do maior potencial de reabilitação em centro de reabilitação face a doentes mais velhos resultando numa diminuição da idade média da população. ${ }^{18} \mathrm{~A}$ prevalência dos tipos/ localização do $\operatorname{AVC}^{19}$ e a evolução média da MIF entre a admissão e a alta durante o processo de reabilitação em regime de internamento vão de acordo ao exposto na literatura. ${ }^{20}$ Apesar da amostra reduzida foi possível também constatar nesta amostra de doentes que MIFs mais elevadas à admissão estiveram associadas a maiores ganhos relativos face aos doentes que foram admitidos com MIFs mais baixas o que vai também de encontro ao descrito na literatura. ${ }^{20} \mathrm{~A}$ distribuição do AVC segundo o sexo continua a ser alvo de diversos estudos, mas a literatura aponta para uma maior prevalência de AVC no sexo feminino a par de uma maior sobrevivência. ${ }^{21,22} \bigcirc$ grau de dependência dos doentes aquando da admissão no centro de reabilitação (70\%) é, como esperado, mais elevado do que o descrito na literatura relativamente à população geral de doentes com AVC. ${ }^{23,24}$ Uma vez que se trata de um centro terciário de reabilitação poderá traduzir um viés na seleção no sentido de se tratar de uma população que possui à partida um maior grau de dependência. Assim, é expectável que mesmo após o internamento num centro de reabilitação a prevalência de dependência de terceira pessoa seja maior do que o descrito na literatura para todos os doentes com sequelas de AVC. Quanto aos Cls, tal como previamente publicado, a maior parte são familiares diretos dos doentes e com uma idade média - 52 anos (26 - 75 anos) - que se aproxima da idade média dos doentes - 62 anos. ${ }^{25-28}$ Neste estudo, a frequência de Cls do sexo masculino foi superior a estudos prévios, ${ }^{25}$ o que poderá ser explicado por dois motivos: tratando-se de um centro de reabilitação poderá existir um viés de seleção em doentes mais graves e dependentes sendo o sexo feminino o sexo mais prevalente; por outro lado, a curta amostra deste estudo pode ser outro fator de viés em relação aos resultados publicados em estudos de maiores dimensões. A distribuição do grau de escolaridade nesta amostra de Cls é atípica e superior à média nacional. ${ }^{29}$ Relativamente à situação profissional $9 \mathrm{Cls}$ já eram previamente reformados e 4 aban- donaram a sua atividade laboral para prestar cuidados o que vai de encontro à literatura disponível: uma parte dos cuidadores está num grupo etário correspondendo à reforma e o impacto na prestação de cuidados obriga, por vezes, ao abandono da atividade laboral30 decorrente do grau de sobrecarga dos Cls. Neste sentido uma das queixas mais frequentes dos Cls é o de não possuírem conhecimentos/competências adequados para lidar com as novas exigências sendo que a primeira fase de adaptação ao doente no domicílio após o AVC é também a fase mais complicada pelas implicações que possuem no quotidiano e na estrutura do seio familiar. ${ }^{3 !}$ No que diz respeito à presença de um $\mathrm{Cl}$ nesta população de doentes é importante referir que um dos critérios para admissão no centro de reabilitação é a existência de um suporte social pós alta previamente definido à admissão. Tendo em conta o elevado grau de dependência dos doentes pós internamento na amostra em estudo e de que em Portugal grande parte desse suporte é prestado pelos Cls é natural que exista um enviesamento no que diz respeito ao número de doentes com Cls. ${ }^{32}$ A ausência de resultados estatisticamente significativos nas relações entre as diferentes variáveis estudadas pode ser explicada pelo baixo poder estatístico da amostra no que diz respeito ao valor total de doentes $(n=22)$. No entanto, é importante referir que os resultados a nível da associação entre as variáveis vão de acordo com o esperado. É expectável que doentes ou Cls mais velhos estejam associados a um maior grau de sobrecarga pelas co-morbilidades/ maior dependência dos primeiros e maior envelhecimento dos segundos e com menor disponibilidade para prestar cuidados. ${ }^{32,33}$ Um fator que também é necessário ter em conta é que o envelhecimento dos doentes é acompanhado pelo envelhecimento dos $\mathrm{Cls}$ com as inerentes dificuldades crescentes na prestação dos cuidados, reforçando a necessidade e pertinência de desenvolver programas que consigam minorar a exigência física e emocional deste tipo de atividade. ${ }^{33}$ Relativamente às características destes Cls importa também relembrar que não são profissionais treinados para prestar cuidados e que executam uma ampla gama de atividades no apoio a estes doentes. $^{32}$ Relativamente à associação negativa entre a MIF à admissão e data de alta e os níveis de sobrecarga importa explicar que esta também vai de encontro ao esperado uma vez que as duas variáveis se comportam de forma antagónica. A níveis mais elevados de MIF e, 
portanto, menor dependência é expectável que existam menores graus de sobrecarga e, portanto, um coeficiente negativo sendo que em ambos os casos não é estatisticamente significativo por possíveis razões já enumeradas anteriormente. ${ }^{34,35}$ Finalmente, parece existir também uma associação entre o grau de sobrecarga e o tempo decorrido desde o AVC bem como o tempo de prestação de cuidados o que poderá ser explicado e enquadrado pelo desgaste acumulado dos $\mathrm{Cl}$ ao longo do tempo. ${ }^{35}$ É, portanto, fundamental educar e dar suporte aos $\mathrm{Cls}$ de doentes no pós AVC de forma a prevenir elevados graus de sobrecarga e consequente burnout: preparar os familiares e Cls para os estados de transição entre as diferentes fases do processo de reabilitação, partilhar informação, educar e promover e aquisição de competências, prestar apoio psicológico e informar doentes e Cls sobre os apoios sociais disponíveis e como aceder aos mesmos. ${ }^{33} \mathrm{~A}$ primeira referência à presença do sobrecarga do $\mathrm{Cl}$ foi em 1986 entre esposas de doentes com patologias crónicas. ${ }^{36}$ Atualmente, o burnout do $\mathrm{Cl}$ é uma entidade bem definida e com claro impacto na prestação de cuidados. ${ }^{37,38}$ Segundo Maslach $^{39}$ o burnout é uma síndrome tridimensional e é composto por três elementos: exaustão emocional| ${ }^{40,41}$ por sentimentos de desgaste e esgotamento dos recursos emocionais; sensação de despersonalização ${ }^{42}$ que origina um distanciamento entre $\circ \mathrm{Cl}$ e o doente até nas tarefas associadas ao ato de cuidar e, finalmente, um terceiro elemento de impacto na realização pessoal com diminuição das expetativas associadas à prestação dos cuidados e diminuição na auto-estima do cuidador. Alguns autores defendem ainda uma distinção entre aquilo que é a sobrecarga subjetiva (a relacionada com os sentimentos e atitudes) e a objetiva (acontecimentos e atividades concretas da vida dos Cls que são alteradas em consequência da prestação de cuidados). ${ }^{43}$ Ao ato de cuidar estão associados diversos aspetos ${ }^{22,44-47}$ positivos e negativos que podem assumir uma preponderância diferente consoante a patologia e o grau de dependência da pessoa cuidada. ${ }^{48}$ Entre $25 \%$ a $45 \%$ dos $\mathrm{Cls}$ confirmam a presença de sintomas compatíveis com sobrecarga nos primeiros 6 meses após o AVC. ${ }^{4}$ Referem diferentes sintomas como ansiedade, frustração, perturbações do sono ou depressão. ${ }^{49} \mathrm{O}$ potencial de reabilitação do doente pode, inclusivamente, estar condicionado pelo grau de sobrecarga do $\mathrm{Cl}^{50}$ Tendo em conta que o reduzido número de $\mathrm{Cls}$ poderá influenciar a obtenção de resultados estatisticamente significativos foi realizada outra análise estatística de acordo com os clusters segundo grau de sobrecarga. Abaixo do QI só existia um $\mathrm{Cl}$ pelo que a nova análise estatística para as variáveis previamente identificadas foi realizada para níveis de sobrecarga entre Q2 e Q3 e acima de Q3. Os resultados foram globalmente sobreponíveis com as seguintes exceções para os níveis de sobrecarga entre Q2 e Q3 sendo que as variáveis tinham valor estaticamente significativo no que diz respeito à relação entre o grau de sobrecarga e a MIF aquando da alta bem como o período decorrido desde o AVC. Já para níveis de sobrecarga acima de Q3 os resultados obtidos continuam a não ser estatisticamente significativos. Quanto ao QSFZ - versão curta existiu uma grande variabilidade no grau de sobrecarga reportada pelos $\mathrm{Cls}$ e o nível médio e a mediana de sobrecarga foi de 17 (Q3), ou seja, metade da amostra apresenta um grau de sobrecarga que representa $25 \%$ dos valores mais elevados possíveis na QSFZ - versão curta 13 o que se traduz num grande impacto e sobrecarga para estes $\mathrm{Cls}$. $\mathrm{O}$ padrão de distribuição do grau de sobrecarga é sobreponível ao publicado na literatura e em diferentes estudos. ${ }^{51-53}$ No entanto, é importante destacar que a maior parte dos Cls estava nesta condição há menos de I ano e que o questionário de sobrecarga foi realizado em média apenas 57 dias após um período de internamento em centro de reabilitação pelo que será de considerar a possibilidade de um agravamento do grau de sobrecarga ao longo do tempo. ${ }^{54}$

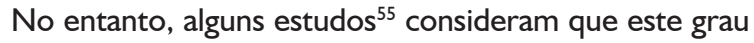
de sobrecarga pode diminuir ao longo do tempo pela adaptação dos $\mathrm{Cls}$ pelo que o grau de sobrecarga pode até ser inferior. Como expectável o maior valor médio nos resultados parciais das diferentes questões dizia respeito ao impacto do ato de cuidar relativamente à situação de dependência e os menores valores médios eram relativos a receio/angústia para com o doente o que poderá estar relacionado com o facto de os cuidadores não quererem culpabilizar o seu familiar (na maior parte dos casos) pela situação de dependência. É importante focar as limitações deste estudo no que diz respeito a alguns aspetos como abranger apenas a experiência de um único centro de reabilitação a nível nacional com o consequente possível enviesamento que daí poderá decorrer seja a nível da população de doentes do próprio centro seja a nível dos critérios de referenciação dos doentes para o mesmo a partir de outras instituições. Desta for- 
ma, a população deste estudo não é representativa da população portuguesa. Por outro lado, apesar de já existirem alguns estudos com a utilização da QSFZ - versão curta em língua portuguesa esta não está validada para a população portuguesa. ${ }^{56,57}$ Tal opção por esta escala prendeu-se com a maior facilidade de utilização da mesma face à original com 22 questões que foi motivo inicial de recusa de muitos dos doentes e $\mathrm{Cl}$ para inclusão neste estudo pela demora na realização das mesmas. Finalmente $\circ \mathrm{n}$ reduzido de doentes limita a obtenção de conclusões estatisticamente significativas. No futuro será relevante integrar outros centros de reabilitação e um maior número de doentes para detalhar o impacto do AVC de doentes previamente internados em centro de reabilitação nos Cls.

\section{Conclusão}

$\mathrm{Na}$ amostra em estudo $50 \%$ dos $\mathrm{Cl}$ apresentava níveis de sobrecarga que se situavam no quartil superior do QSFZ - versão curta e que traduz o grande impacto nos Cls de doentes internados por AVC em centro de reabilitação. Apesar do reduzido número de doentes foi possível obter relações estaticamente significativas na análise por clusters de sobrecarga no que diz respeito aos Cls entre Q2 e Q3 e à relação entre o grau de sobrecarga e a MIF aquando da alta e o tempo decorrido desde - AVC. A inexistência de outras relações estaticamente significativas poderá ser explicada pelo enviesamento da população e / ou reduzida amostra da população do estudo que não é representativa da população portuguesa. Estes resultados obtidos não vão totalmente de acordo ao impacto expectável nos $\mathrm{Cls}$, mas tal poderá ser justificado pelas razões acima enunciadas ou por se tratar de uma população de um centro de reabilitação onde os Cls integram o plano de reabilitação a nível de ensinos e acompanhamento ao doente internado. É também importante destacar a originalidade deste artigo no que diz respeito à análise do nível de sobrecarga dos $\mathrm{Cls}$ de doentes com AVC previamente internados em centro de reabilitação uma vez que não foi encontrado pelos autores nenhum outro artigo semelhante realizado em Portugal. No futuro será útil integrar outros centros de reabilitação e maior número doentes para melhor caracterização da sobrecarga dos $\mathrm{Cls}$ de doentes com AVC internados em centros de reabilitação.

\section{Responsabilidades Éticas}

Conflitos de Interesse: Os autores declaram a inexistência de conflitos de interesse na realização do presente trabalho.

Fontes de Financiamento: Não existiram fontes externas de financiamento para a realização deste artigo.

Confidencialidade dos Dados: Os autores declaram ter seguido os protocolos da sua instituição acerca da publicação dos dados de doentes.

Proteção de Pessoas e Animais: Os autores declaram que os procedimentos seguidos estavam de acordo com os regulamentos estabelecidos pelos responsáveis da Comissão de Investigação Clínica e Ética e de acordo com a Declaração de Helsínquia da Associação Médica Mundial.

Proveniência e Revisão por Pares: Não comissionado; revisão externa por pares.

\section{Ethical Disclosures}

Conflicts of interest: The authors have no conflicts of interest to declare.

Financing Support: This work has not received any contribution, grant or scholarship

Confidentiality of Data: The authors declare that they have followed the protocols of their work center on the publication of data from patients.

Protection of Human and Animal Subjects: The authors declare that the procedures followed were in accordance with the regulations of the relevant clinical research ethics committee and with those of the Code of Ethics of the World Medical Association (Declaration of Helsinki).

Provenance and Peer Review: Not commissioned; externally peer reviewed.

\section{References / Referências}

1. Portugal, Serviço Nacional de Saúde. Retratos da Saúde. Lisboa: SNS; 2018.

2. Johnson W, Onuma O, Owolabi M, Sachdev S. Stroke: a global response is needed. Bull World Health Organ. 2016;94:634-634A. doi: 10.2471/BLT.16.181636.

3. Pucciarelli G, Ausili D, Rebora P, Arisido MW, Simeone S, Alvaro $R$, et al. Formal and informal care after stroke: $A$ longitudinal analysis of survivors' post rehabilitation hospital discharge. J Adv Nurs. 2019;75:2495-505. doi:10.1111/ jan.13998

4. Zhu W, Jiang Y. A Meta-analytic study of predictors for informal caregiver burden in patients with stroke. J Stroke Cerebrovasc Dis. 2018;27:3636-46. doi:10.1016/j.jstrokecerebrovasdis.2018.08.037

5. Rocha Pereira H. Subitamente Cuidadores Informais Dando Voz(Es) Às Experiências Vividas. Lisboa: Lusociência; 2013.

6. Tsai YC, Pai HC. Burden and cognitive appraisal of stroke survivors' informal caregivers: an assessment of depression model with mediating and moderating effects. Arch Psychiatr Nurs. 2016;30:237-43. doi:10.1016/j.apnu.2015.11.007

7. Ganapathy V, Graham GD, Dibonaventura MD, Gillard PJ, Goren A, Zorowitz RD. Caregiver burden, productivity loss, and indirect costs associated with caring for patients with poststroke spasticity. Clin Interv Aging. 2015;10:1793-802. doi:10.2147/CIA.S91123

8. Sousa L, Figueiredo D, Cerqueira M. Envelhecer em família: cuidados familiares na velhice. Porto: Ambar; 2004.

9. Maslach C, Jackson SE, Leiter MP. Maslach Burnout Inventory. 3rd ed. Palo Alto: Consulting Psychologists Press; 1996.

10. Schaufeli WB, Taris TW. The conceptualization and measurement of burnout: Common ground and worlds apart. Work Stress. 2005;19: 256-62. doi:10.1080/02678370500385913

11. Mikolajczak M, Roskam I. A theoretical and clinical framework for parental burnout: The balance between risks and resources (BR2). Front Psychol. 2018;9:886. doi:10.3389/ fpsyg.2018.00886

12. Zarit SH, Zarit JM. The memory and behavior problems checklist-1987R and the burden interview (technical report). 
Pennsylvania: Univ Park Pennsylvania State Univ; 1987.

13. Bédard M, Molloy DW, Squire L, Dubois S, Lever JA, O'donnell M. The Zarit Burden Interview: A new short version and screening version. Gerontologist. 2001;41:652-7. doi:10.1093/geront/41.5.652

14. Yousufuddin $M$, Young N. Aging and ischemic stroke. Aging. 2019;11:2542-4. doi:10.18632/aging.101931

15. Kissela BM, Khoury JC, Alwell K, Moomaw CJ, Woo D, Adeoye $\mathrm{O}$, et al. Age at stroke: Temporal trends in stroke incidence in a large, biracial population. Neurology. 2012;79:1781-7. doi:10.1212/WNL.0b013e318270401d

16. Kelly-Hayes M. Influence of age and health behaviors on stroke risk: Lessons from longitudinal studies. J Am Geriatr Soc. 2010;58:S325. doi:10.1111/j.1532-5415.2010.02915.x

17. Ekker MS, Verhoeven JI, Vaartjes I, Van Nieuwenhuizen KM, Klijn CJ, De Leeuw FE. Stroke incidence in young adults according to age, subtype, sex, and time trends. Neurology. 2019;92:e2444-54. doi:10.1212/WNL.0000000000007533

18. Janzen S, Mirkowski M, Mclntyre A, Mehta S, Iruthayarajah $\mathrm{J}$, Teasell R. Referral patterns of stroke rehabilitation inpatients to a model system of outpatient services in Ontario, Canada: A 7-year retrospective analysis. BMC Health Serv Res. 2019;19:399. doi:10.1186/s12913-019-4236-5

19. Benjamin EJ, Blaha MJ, Chiuve SE, ushman M, Das SR, Deo R, et al. Heart Disease and Stroke Statistics'2017 Update: A Report from the American Heart Association. Circulation. 2017;135:e146-e603.. doi:10.1161/CIR.0000000000000485

20. Ng YS, Stein J, Ning MM, Black-Schaffer RM. Comparison of clinical characteristics and functional outcomes of ischemic stroke in different vascular territories. Stroke. 2007;38:230914. doi:10.1161/STROKEAHA.106.475483

21. Dehlendorff $C$, Andersen KK, Olsen TS. Sex disparities in stroke: women have more severe strokes but better survival than men. J Am Heart Assoc. 2015;4:e001967. doi:10.1161/ JAHA.115.001967

22. Washington KT, Pike KC, Demiris G, Parker Oliver D, Albright DL, Lewis AM. Gender Differences in Caregiving at End of Life: Implications for Hospice Teams. J Palliat Med. 2015:1048-53. doi:10.1089/jpm.2015.0214

23. Fernandes TG, Goulart AC, Santos-Junior WR, Alencar $A P$, Benseñor IM, Lotufo PA. Educational levels and the functional dependence of ischemic stroke survivors. Cad Saude Publica. 2012;28:1581-90. doi:10.1590/s0102$311 \times 2012000800016$

24. Ullberg T, Zia E, Petersson J, Norrving B. Changes in functional outcome over the first year after stroke: An observational study from the Swedish stroke register. Stroke. 2015 ;46:389-94. doi:10.1161/STROKEAHA.114.006538

25. Lui MHL, Ross FM, Thompson DR. Supporting family caregivers in stroke care: A review of the evidence for problem solving. Stroke. 2005;36:2514-22. doi:10.1161/01. STR.0000185743.41231.85

26. REBELO ÂA. Prestadores de cuidados informais a idosos com 80 e mais anos, na freguesia de Moreira da Maia. Geriatria. 1996.

27. Escuredo Rodríguez B, Díaz Alvarez E, Pascual Cortés O. Cuidadores informales. Necesidades y ayudas. Rev Enferm. 2001;24:183-9

28. RICARTE LFCS. Sobrecarga do cuidador informal de idosos dependentes no Concelho da Ribeira Grand. 2009.

29. Instituto Nacional de Estatística. Censos 2011. Lisboa: INE; 2011.

30. Caregiving NA for. Caregiving in the U.S. 2015

31. Mors NP, Sørensen L V, Therkildsen ML. The impact on the relatives of psychiatric patients admitted for the first time. Ugeskr Laeger. 1993.

32. União Europeia. Informal Care in Europe - Exploring Formalisation, Availability and Quality.; 2018.

33. Mores G, Whiteman R, Knobl P, Ploeg J, Cahn M, Klaponski $L$, et al. Pilot evaluation of the family informal caregiver stroke self-management program. Can J Neurosci Nurs. 2013;35:18-26.
34. Ostwald SK, Bernal MP, Cron SG, Godwin KM. Stress experienced by stroke survivors and spousal caregivers during the first year after discharge from inpatient rehabilitation. Top Stroke Rehabil. 2009;16:93-104. doi:10.1310/tsr1602-93

35. Oliva-Moreno J, Peña-Longobardo LM, Mar J, Soulard S, Gonzalez-Rojas N, Becerra V, et al. Determinants of informal care, burden, and risk of burnout in caregivers of stroke survivors the CONOCES Study. Stroke. 2018;49:140-6. doi:10.1161/STROKEAHA.117.017575

36. Ekberg JY, Griffith N, Foxall MJ. Spouse burnout syndrome. J Adv Nurs. 1986;11:161-5. doi: 10.1111/j.1365-2648.1986. tb01234.x.

37. Angermeyer MC, Bull N, Bernert S, Dietrich S, Kopf A. Burnout of caregivers: a comparison between partners of psychiatric patients and nurses. Arch Psychiatr Nurs. 2006;20:15865. doi: 10.1016/j.apnu.2005.12.004.

38. Thorson-Olesen SJ, Meinertz N, Eckert S. Caring for aging populations: examining compassion fatigue and satisfaction. J Adult Dev. 2019; 26: 232-40.. doi:10.1007/s10804018-9315-z

39. Maslach C. Burnout the Cost of Caring.; 1982.

40. Thompson R, Kerr M, Glynn M, Linehan C. Caring for a family member with intellectual disability and epilepsy: practical, social and emotional perspectives. Seizure. 2014;23:856-63. doi: 10.1016/j.seizure.2014.07.005.

41. Goodwin J, McCormack L, Campbell LE. "You don't know until you get there": The positive and negative "lived" experience of parenting an adult child with 22q11.2 deletion syndrome. Health Psychol. 2017;36:45-54. doi: 10.1037/ hea0000415.

42. Cross AJ, Garip G, Sheffield D. The psychosocial impact of caregiving in dementia and quality of life: a systematic review and meta-synthesis of qualitative research. Psychol Health. 2018;33:1321-42. doi: 10.1080/08870446.2018.1496250.

43. Rolo L. Sobrecarga e Satisfação com a Vida. 2009.

44. Fátima Ferreira, Adalgiza Pinto AL. Validação da escala de Zarit: sobrecarga do Cuidador em cuidados paliativos domiciliários, para população portuguesa. Cad Saúde.

45. Vellone E, Fida R, Cocchieri A, Sili A, Piras G, Alvaro R. Positive and negative impact of caregiving to older adults: a structural equation model. Prof Inferm. 2011;64:237-48.

46. Pinquart M, Sörensen S. Gender differences in caregiver stressors, social resources, and health: an updated metaanalysis. J Gerontol B Psychol Sci Soc Sci. 2006;61:P33-45. doi: 10.1093/geronb/61.1.p33.

47. Redecker, C., Reig, J., Carrión, T., Martinez, S., Armayones, M., \& McCarthy D. The potential of ICT in supporting domiciliary care in Spain. Jt Res Cent. 2010.

48. Oliveira AL. Cuidados Informais ao Idoso Dependente: Motivos e gratificações. 2009.

49. Walker MF, Thomas SA, Whitehead PJ, Condon L, Fisher RJ, Kontou E, et al. Biopsychosocial Intervention for Stroke Carers (BISC): protocol for a feasibility randomised controlled trial (RCT). BMJ Open. 2017;7:e018309. doi: 10.1136/bmjopen-2017-018309.

50. Olai L, Borgquist L, Svärdsudd K. Life situations and the care burden for stroke patients and their informal caregivers in a prospective cohort study. Ups J Med Sci. 2015;120:290-8. doi: 10.3109/03009734.2015.1049388.

51. Oliva-Moreno J, Peña-Longobardo LM, Mar J, Masjuan J, Soulard S, Gonzalez-Rojas N, et al; CONOCES Investigators Group. Determinants of Informal Care, Burden, and Risk of Burnout in Caregivers of Stroke Survivors: The CONOCES Study. Stroke. $2018 ; 49: 140-6$. doi: 10.1161/STROKEAHA.117.017575.

52. Hu P, Yang $Q$, Kong L, Hu L, Zeng L. Relationship between the anxiety/depression and care burden of the major caregiver of stroke patients. Medicine. 2018;97:e12638. doi:10.1097/MD.0000000000012638

53. Pereira RA, dos Santos EB, Fhon JRS, Marques S, Rodrigues RP. Burden on caregivers of elderly victims of cerebrovascular accident. Rev da Esc Enferm. 2013;47:182-8. 
doi:10.1590/S0080-62342013000100023

54. Pont W, Groeneveld I, Arwert H, Meesters J, Mishre RR, Vliet Vlieland T, et al. Caregiver burden after stroke: changes over time? Disabil Rehabil. 2020;42:360-7. doi:10.1080/096 38288.2018.1499047

55. Zarit SH, Todd PA, Zarit JM. Subjective burden of husbands and wives as caregivers: a longitudinal study. Gerontologist. 1986;26:260-6. doi:10.1093/geront/26.3.260

56. Gratão ACM, Brigola AG, Ottaviani AC, Luchesi BM, Souza ÉN, Rossetti ES,et al. Brief version of Zarit Burden
Interview (ZBI) for burden assessment in older caregivers. Dement Neuropsychol. 2019;13:122-9. doi:10.1590/198057642018dn13-010015

57. Ballesteros J, Santos B, González-Fraile E, Muñoz-Hermoso P, Domínguez-Panchón Al, Martín-Carrasco M. Unidimensional 12-item zarit caregiver burden interview for the assessment of dementia caregivers' burden obtained by item response theory. Value Heal. 2012;15:1141-7. doi:10.1016/j. jval.2012.07.005 